\title{
Mission and System Design for the Manipulation of PHOs with Space-borne Lasers
}

\author{
Nicolas Thiry, Massimiliano Vasile \\ Department of Mechanical \& Aerospace Engineering, \\ University of Strathclyde, Glasgow, UK \\ nicolas.thiry@ strath.ac.uk, massimiliano.vasile@strath.ac.uk \\ Emanuele Monchieri \\ Future Programs Division, \\ Airbus Defence \& Space, Gunnels Wood Road, Stevenage,UK \\ emanuele.monchieri@airbus.com,
}

\begin{abstract}
Owing to their ability to move a target in space without requiring propellant, laser-based deflection methods have gained attention among the research community in the recent years. With laser ablation, the vaporized material is used to push the target itself allowing for a significant reduction in the mass requirement for a space mission. Specifically, this paper addresses two important issues which have remained unanswered by previous studies: the impact of the tumbling motion of the target as well as the impact of the finite thickness of the material ablated in the case of a space debris. We developed an analytical model based on energetic considerations in order to predict the efficiency range theoretically allowed by a $\mathrm{CW}$ laser deflection system operating under the plasma formation threshold and in absence of the two aforementioned issues. A numerical model was then developed to solve the transient heat equation in presence of vaporization and melting and assess the efficiency reduction due to the unsteadiness induced by the PHO's tumbling motion. The model was translated to handle the case where the target is a piece of space debris by considering specific materials such as aluminum and titanium alloys or even carbon fiber and by adapting the finite size of the computational domain along with the propagation of the ablation front. From the results of this later model, pulsed lasers system appear better suited to answer the needs of a space debris de-orbiting laser system rather than CW lasers. An empirical ablation threshold is also found that establishes a direct relation between the pulse duration or the heating time (CW case), the delivered flux and the properties of the material. Derived from theoretical consideration, this threshold matches well with the predictions of our numerical model. Moreover, the numerical results are found to agree with published data of thrust coupling coefficient on target made of aluminium and titanium alloys. In the second part of the paper, we coupled our thrust model within an orbit propagator and considered several redirect scenarios for the case of a small $(56 \mathrm{~m})$ and a larger $(100 \mathrm{~m})$ asteroid as well as an $8 \mathrm{~T}$ defunct satellite currently orbiting in a sun-synchronous orbit at a $765 \mathrm{~km}$ altitude. Based on the results, realistic mission architectures are explored for these scenarios. Within this last section, the paper also highlights the advantages offered in term of redundancy and scalability by techniques such as beam combining or formation flying. Our results indicate that a a medium class mission carrying a $\mathrm{CW}$ laser system able to generate $2.4 \mathrm{~kW}$ of output power could ensure the deflection of a $56 \mathrm{~m}$ asteroid while a formation of such spacecrafts could also achieve the deflection of a larger threat. Our preliminary results for the debris case also indicates that an actively Q-switched diode-pumped solid state laser (DPSSL) with $3 \mathrm{~kW}$ of output power would reduce the altitude of Envisat in less than 500 days.
\end{abstract}

978-1-4673-7676-1/16/\$31.00 (c)2016 IEEE
TABle of Contents

1. INTRODUCTION.............................1

2. Mechanical Coupling During the Ablation

PROCESS .......................................2

3. UnCooperative Target Redirect Missions ..6

4. System Design for an ASTeroid Redirect

MISSION .....................................9

5. ConCLUSION ................................... 11

ACKNOWLEDGMENTS ......................... 11

REFERENCES ................................... 11

BIOGRAPHY $\ldots \ldots \ldots \ldots \ldots \ldots \ldots \ldots \ldots \ldots \ldots \ldots, 12$

\section{INTRODUCTION}

The recent years have seen an increasing public awareness over the catastrophic consequences that would arise from the collision between an asteroid and the Earth. Popularized by science-fiction movies such as Deep Impact or Armaggedon, the usual fear is that of a large impactor $(10 \mathrm{~km})$ causing global-scale devastation. While evidences, such as the Chicxulub crater, points out that such events indeed happened in the past and will happen again, the impact frequency of a $10 \mathrm{~km}$ bolide is only 1 every 100 millions years. On the other hand, smaller bodies, such as the asteroid responsible for the Chelyabinsk event two years ago are much more frequent and therefore more likely to represent an immediate threat to our terrestrial assets. As an example, the asteroid that struck Tunguska in 1908 was only about 60 meters across, yet knocked down 80 million trees over an area of 2000 square kilometers. Thus, there is a clear rationale to develop technologies that are able to manipulate precisely the trajectory of these small asteroids.

Generally speaking, deflection methods can be divided into two main categories: impulsive and slow-push. Impulsive techniques are generally modelled with an instantaneous change of momentum given by, for example, a nuclear explosion (nuclear interceptor) or the hypervelocity impact of a spacecraft (kinetic impactor) with the asteroid. Slow-push methods, on the other hand, allow for a more controllable deflection manoeuvre by exerting a small continuous and controllable force on the asteroid over an extended period of time. Also, downscaling the concepts for slow-push deflection methods would in principle allow to consider also their use to the case the target is a man-made space debris rather than an asteroid, something not feasible with impulsive techniques. 
Over the past years many slow-push concepts have been proposed and studied at various degrees of accuracy. Many of them are based on the use of electric propulsion and therefore require a dedicated propulsion system and propellant to generate the necessary deflection. In contrast, slowpush ablation-based methods (such as direct solar or laser ablation) aim at exploiting the material the asteroid is made of, to generate the required thrust. In the work of Kahle[1] and Vasile[2], however, it was shown that the contamination of the solar collectors, severely limits the effectiveness of direct solar ablation. On the other hand, as demonstrated by Vasile[3], if the deflection is achievable in a given limit time, laser ablation techniques require a lower mass into space than electric propulsion methods. The use of lasers, compared to directly focusing the light of the Sun, implies higher conversion losses but has the distinctive advantage to provide high light intensity at lower power and longer distance from the target.

Several mission concepts using in-space laser ablation have been investigated in the past using a single laser powered by nuclear reactors [4] or swarms of lasers powered by the Sun [5]. A swarm of spacecraft flying in formation in the proximity of the target asteroid allows combining multiple beams to produce a high thrust level while keeping the power and thermal systems to a manageable size and complexity on each individual spacecraft. In addition, the swarm offers a more reliable system [6] in the case of failure of a single spacecraft and a more controllable thrust vector as ablating different parts of the asteroid surface will reduce the uncertainty on the direction of the resultant thrust vector.

Recently a study supported by the European Space Agency, demonstrated the feasibility of laser ablation at changing the orbital velocity of a 130 tons S-type asteroid by $1 \mathrm{~m} / \mathrm{s}$ in less than 1 year. The concept, called Light Touch [7], was considering the use of a commercial fibre laser, installed on a $453 \mathrm{kgs}$ spacecraft, and requiring between $460 \mathrm{~W}$ and $860 \mathrm{~W}$ of input power.

In this paper, we study the efficiency of deflection methods using laser ablation both for asteroids and space debris. The key metric to assess the performance is given by the socalled thrust-coupling coefficient, which is given by the ratio between the thrust magnitude and the optical power required to generate that thrust. The paper is divided in 3 sections. In section 1 , the principle underlying the ablation physics and the way the thrust is formed from the vaporized plume is reviewed. A precise numerical model is then developed for an asteroid or thin debris materials such as aluminum or titanium alloys and carbon fiber. These results suggest that pulsed lasers system are better suited to answer the needs of a space debris de-orbiting laser system rather than CW lasers. An empirical ablation threshold is also found that links the pulse duration (or the mean heating time in the tumbling CW case), the delivered flux and the properties of the material. Derived from theoretical consideration, this threshold matches well with the predictions of our numerical model. Moreover, the numerical results are found to agree with published data of thrust coupling coefficient on target made of aluminium and titanium alloys. In the second part of the paper, we couple this thrust model within an orbit propagator and considered several redirect scenarios for the case of a small $(56 \mathrm{~m})$ to larger $(100 \mathrm{~m})$ asteroid and an $8 \mathrm{~T}$ defunct satellite currently orbiting in a sun-synchronous orbit. Based on the results, realistic mission architectures are finally explored for these scenarios. Within this last section, the paper also investigates the advantages offered in term of redundancy and scalability by techniques such as beam combining or formation flying. To conclude, we indicate that a medium class mission carrying a $5 \mathrm{~kW}$ laser could ensure the deflection of a $56 \mathrm{~m}$ asteroid while a formation of such spacecrafts could also achieve the deflection of a larger $(100 \mathrm{~m})$ threat.

\section{Mechanical Coupling During the Ablation Process}

This section reviews the principle underlying the laser ablation deflection method. The general physics is explained in the first subsection while a numerical model is derived in the next subsection to demonstrate the reduced efficiency linked with the rotation of the target. The same model is then used to predict the efficiency of pulsed wave laser for space debris and is compared with existing experimental data for titanium and aluminium alloys. The last subsection details the contamination model and some parameters derived from previous experimental work carried in our lab using a $100 \mathrm{~W}$ CW fiber-coupled laser and asteroid analogue samples [8].

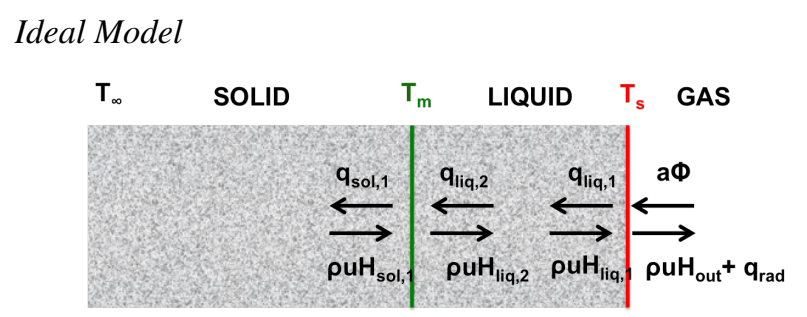

Figure 1: Energy transport during the ablation process

We consider in this work a rocky S-type asteroid essentially made of forsterite as well as several materials that can be found in space debris such as aluminium and titanium allows and carbon fibers. Assumed properties can be reviewed in table 1 . Note that the values of thermal conductivity, density and absorptivity are effective values in the temperature range of interest. In particular, the thermal conductivity of liquid aluminium alloys and metals can be less than half of the one at room temperature [9][10]. When available, high temperature electric resistivity measurements can be used to reconstruct the thermal conductivity using the Wiedemann-Franz law [11].

With the notable exception of carbon, the surface temperature reached during the ablation process is substantially higher than the triple point of the substance considered. Therefore the ablated material undergoes successive phase transformations before reaching the vapour state and very thin layer of molten material is formed under the ablation front. A simple energy balance allows us to express the energy absorbed by thermal conduction through the different interfaces and to derive the continuity relation along the vaporization and melting fronts:

$$
\begin{aligned}
& q_{l i q, 1}=A \Phi-q_{r a d}-\rho u_{v} E_{v} \\
& q_{l i q, 2}=q_{s o l, 1}+\rho u_{m} E_{m}
\end{aligned}
$$

The mass flow during the vaporization process is a consequence of the thermodynamical non-equilibrium at the interface. As they vaporize, the molecules acquire a net translational velocity component through collisions at the molecular level, resulting in their distribution function becoming a shifted Maxwellian distribution. The finite layer through which the drift velocity can be acquired is called the 
Table 1: Material properties considered for the computations

\begin{tabular}{lllllll}
\hline Quantity & Symbol & Forsterite & Al2024 - T3 & Ti 6Al-4V & Carbon Fiber & Unit \\
\hline Density (sol - liq) & $\rho$ & 3280 & $2780-1800$ & $4506-4110$ & 1780 & $\mathrm{~kg} / \mathrm{m}^{3}$ \\
Thermal Conductivity & $k$ & 2 & 100 & 16 & 20 & $\mathrm{~W} \cdot \mathrm{m}^{-1} \cdot \mathrm{K}^{-1}$ \\
Heat Capacity (liq) & $c_{l}$ & 1464 & 1177 & 984 & - & $\mathrm{J} \cdot \mathrm{kg}^{-1} \cdot \mathrm{K}^{-1}$ \\
Heat Capacity (solid) & $c_{s}$ & 1264 & 1063 & 702 & 2000 & $\mathrm{~J} \cdot \mathrm{kg}^{-1} \cdot \mathrm{K}^{-1}$ \\
Vaporization Enthalpy & $E_{v}$ & $14.16[12]$ & 10.53 & 8.85 & 59.33 & $\mathrm{MJ} / \mathrm{kg}$ \\
Melting Enthalpy & $E_{m}$ & 0.508 & 0.397 & 0.295 & - & $\mathrm{MJ} / \mathrm{kg}$ \\
reference temperature & $T_{r e f}$ & 3000 & 2790 & 3560 & 3915 & $\mathrm{~K}$ \\
reference pressure & $p_{r e f}$ & 4448.9 & $1 \mathrm{e}+5$ & $1 \mathrm{e}+5$ & $1 \mathrm{e}+5$ & $\mathrm{pa}$ \\
Melting point & $T_{m}$ & 2171 & 925 & 1941 & 4600 & $\mathrm{~K}$ \\
Gas Constant & $\mathrm{R}^{*}$ & 208 & 308 & 173 & 692 & $\mathrm{~J} \cdot \mathrm{kg}^{-1} \cdot \mathrm{K}^{-1}$ \\
Heat ratio (gas) & $\gamma$ & 1.26 & 1.67 & 1.67 & 1.67 & - \\
Emissivity & $\epsilon$ & 0.9 & 0.07 & 0.19 & 0.9 & - \\
Absorptivity $(1.06 \mu \mathrm{m})$ & $A$ & 0.8 & $0.2(350 \mathrm{~nm}[13])$ & $0.4(350 \mathrm{~nm}[10])$ & $0.6[14]$ & - \\
Rest temperature & $T_{\infty}$ & 298 & 298 & 298 & 298 & $\mathrm{~K}$ \\
\hline
\end{tabular}

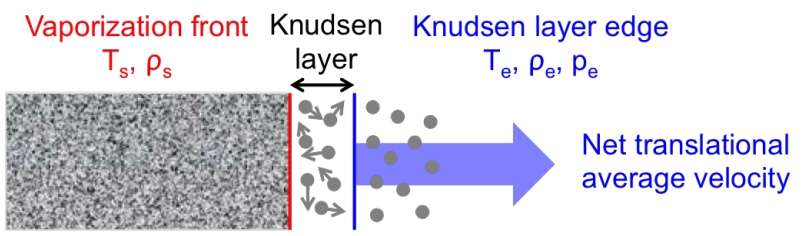

Figure 2: The Knudsen Layer

Knudsen layer and is treated in the model as a gas-dynamic discontinuity. The jump conditions have been investigated by Knight[15] and are given in equation 3 :

$$
\begin{aligned}
m & =\sqrt{\frac{\gamma}{2}} M_{e}=\frac{v_{e}}{\sqrt{2 R^{*} T_{e}}} \\
\frac{T_{e}}{T_{s}} & =\left[\sqrt{1+\pi\left(\frac{\gamma-1}{\gamma+1} \frac{m}{2}\right)^{2}}-\sqrt{\pi} \frac{\gamma-1}{\gamma+1} \frac{m}{2}\right]^{2} \\
\frac{\rho_{e}}{\rho_{s}} & =\sqrt{\frac{T_{s}}{T_{e}}}\left[\left(m^{2}+\frac{1}{2}\right) e^{m^{2}} \operatorname{erfc}(m)-\frac{m}{\sqrt{\pi}}\right] \\
& +\frac{1}{2} \frac{T_{s}}{T_{e}}\left[1-\sqrt{\pi} m e^{m^{2}} \operatorname{erfc}(m)\right]
\end{aligned}
$$

In this expression, $\mathrm{M}_{e}$ represents the local Mach number on the edge of the Knudsen layer and is dependent on the environment downstream. In vacuum, the flow reaches the sonic limit so that the gas-dynamic part can be uncoupled from the thermal problem. The mass flow can thus directly be computed from equation 3 once the temperature $\mathrm{T}_{s}$ of the interface is known:

$$
\rho u\left(T_{s}\right)=\rho_{e} \sqrt{\gamma R^{*} T_{e}}=\alpha_{v} \frac{p_{s}}{\sqrt{2 \pi R^{*} T_{s}}}
$$

Where the RHS is equivalent to the well-known HertzKnudsen-Langmuir formula taking an evaporation fraction $\alpha_{v}$ of

$$
\alpha_{v}=\frac{\rho_{e}}{\rho_{s}} \sqrt{\frac{2 \pi \gamma T_{s}}{T_{e}}}
$$

The liquid near the interface is on the other hand assumed to be near equilibrium [15]. A Clausius-Clapeyron relation together with the law of perfect gas is used to obtain the dependency between $\rho_{s}$ and $T_{s}$ in the gas:

$$
\begin{aligned}
p_{s} & =\rho_{s} R^{*} T_{s} \\
\log \left(\frac{p_{s}}{p_{\text {ref }}}\right) & =\frac{E_{v}}{R}\left(\frac{1}{T_{\text {ref }}}-\frac{1}{T_{s}}\right)
\end{aligned}
$$

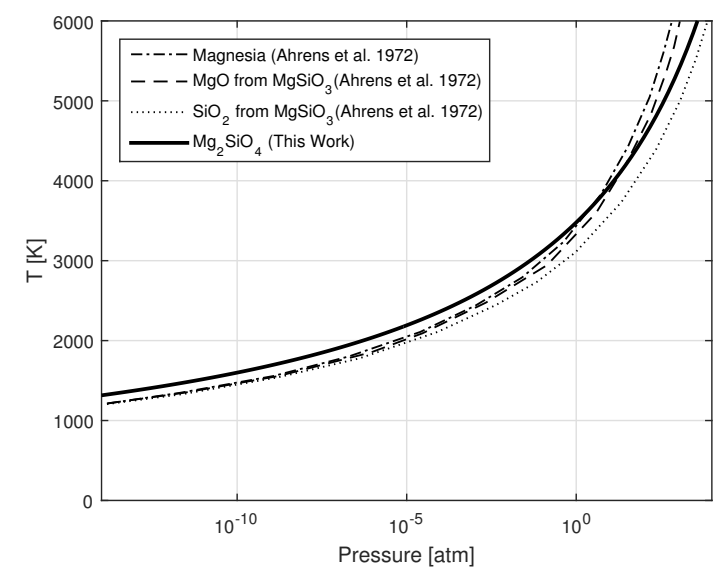

Figure 3: Equilibrium pressure $\mathrm{p}_{s}$ of Forsterite as a function of the surface temperature $\mathrm{T}_{s}$

When sufficient time is allowed to heat the target, the ablation proceeds in a steady-state regime. Therefore, the internal energy becomes invariant with respect to time and the heat conducted through the ablation front $q_{l i q, 1}$ is balanced by the heat required to heat the flow of material from the rest temperature until the surface temperature. In this regime, an implicit relation can be found to link the interface temperature to the laser flux $\Phi$ :

$$
\begin{aligned}
A \Phi & =\epsilon \sigma\left(T_{s}^{4}-T_{\infty}^{4}\right) \\
& +\rho u\left(E_{v}+E_{m}+c_{l}\left(T_{s}-T_{m}\right)+c_{s}\left(T_{m}-T_{\infty}\right)\right)
\end{aligned}
$$

Determining the surface temperature, the net force per unit area $\mathrm{p}_{\text {eff }}$ under the spot can be computed by summing the 
rate of momentum change to the pressure at the edge of the Knudsen layer:

$$
\begin{aligned}
p_{e f f} & =p_{e}+\rho_{e} c_{e}^{2} \\
& =(\gamma+1) p_{e}
\end{aligned}
$$

The thrust coupling $\mathrm{C}_{m}$ is defined as the ratio of the force exerted by the power injected:

$$
C_{m}=\frac{p_{e f f}}{\Phi}
$$

Using this procedure, figure 4 shows the thrust coupling coefficient computed for Forsterite and several materials that can be found on space debris. The values predicted seem to stall between 10 to $100 \mu N / \mathrm{W}$, which is consistent with the work of Phipps[16]. When transient effects can be neglected, we see that the ablation threshold is essentially driven by the absorptivity-emissivity ratio of the material, with a high ratio (aluminium alloys) favoring the ablation onset. On the other hand, the plateau value reached by the coupling coefficient is essentially linked with the absorptivity-vaporization enthalpy ratio of the material. This explains why Forsterite, which has a much higher absorptivity than the other materials, has a better coupling coefficient than carbon, titanium and aluminium alloys. On the other hand, Carbon has a higher absorptivity than aluminium but also requires a much higher Vaporization energy.

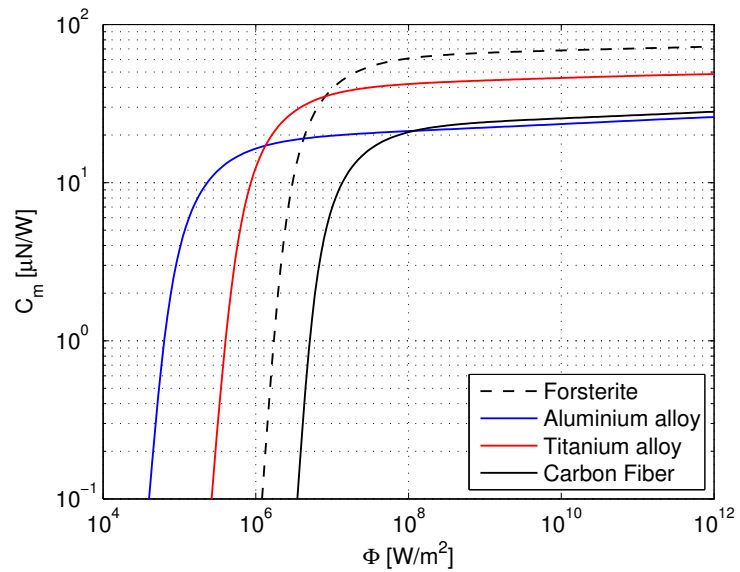

Figure 4: Thrust coupling as a function of the flux for Forsterite and several materials found on space debris

\section{Model for a Moving Target}

It was assumed in the previous section that the time allowed to heat a given point at the surface is long enough so that transient effects can be neglected. In practice, the tumbling motion of the target induces a limitation on the time available to heat a given point at its surface. Considering the relative velocity of the ablated surface respectively to the laser beam $\mathrm{v}_{\text {rel }}$ and the diameter $\phi$ of the laser beam, a mean heating time $\tau$ can be computed as

$$
\tau=\frac{\pi}{4} \frac{\phi}{v_{r e l}}
$$

Therefore, in order to understand how the tumbling motion affects the ablation process, the transient heat equation needs to be solved. An enthalpy formulation with a frame attached to the moving ablation front is selected in Eq.(12), allowing to handle the different phase transitions in a more convenient way:

$$
\frac{\partial(\rho H)}{\partial t}=-\frac{\partial q}{\partial z}+\frac{\partial(\rho u H)}{\partial z}
$$

In this equation, $\mathrm{u}$ is the recession speed of the ablation front and $q$, the heat flux, which is expressed through the common Fourier law $q=-k \frac{d T}{d z}$. Eq.(12) can be solved numerically by taking $N$ control volumes along the depth direction $z$ and applying the conservation of the enthalpy to each of them as follows:

$$
\frac{d(\rho H)_{i}}{d t}=-\frac{q_{i+1 / 2}-q_{i-1 / 2}}{\Delta z}+u\left(T_{1}\right) \frac{(\rho H)_{i+1}-(\rho H)_{i}}{\Delta z}
$$

The fluxes are then computed from:

$$
\begin{aligned}
q_{i+1 / 2} & =-k \frac{T_{i+1}-T_{i}}{\Delta z} \\
q_{i-1 / 2} & =-k \frac{T_{i}-T_{i-1}}{\Delta z}
\end{aligned}
$$

The temperature is recovered at each time step from the enthalpy which is for convenience defined equal to 0 at the melting temperature:

$$
\begin{array}{rr}
T_{i}=T_{m}+\frac{H_{i}}{c_{s}} & \text { if } H_{i} \leq 0 \\
T_{m} & \text { if } 0<H_{i}<E_{m} \\
& T_{m}+\frac{H_{i}-E_{m}}{c_{l}}
\end{array}
$$

The boundary conditions are then introduced through:

$$
\begin{aligned}
q_{1-1 / 2} & =A \Phi-q_{\text {rad }}\left(T_{1}\right)-\rho u\left(T_{1}\right) E_{v} \\
q_{N+1 / 2} & =-k \frac{T_{\infty}-T_{N}}{\Delta z}
\end{aligned}
$$

Equation (13) is integrated in Matlab ${ }^{(c)}$ using ode23t which is suitable for moderately stiff problems. The time-dependent thrust coupling coefficient $C_{m}^{\text {tr }}$ is recovered from the surface temperature at each time-step and an effective thrust coupling coefficient $C_{m}$ is simply obtained by averaging its value over the exposition period:

$$
C_{m}=\frac{.87}{\tau} \int_{0}^{\tau} C_{m}^{\mathrm{tr}} d t
$$

In which the .87 factor accounts for the losses due to the noncylindrical energy density of $\mathrm{TEM}_{00}$ mode [17]. The results are represented for Forsterite in figure 5 and a $1 \mathrm{~mm}$ thin panel of aluminium alloy on figure 6 .

It appears that the ablation threshold is shifted towards higher flux following a trend line in logarithmic scale such that $(\Phi \sqrt{\tau})_{\text {Threshold }}$ is a constant. If one neglects the melting and vaporization processes, it can in fact be shown [18] that the temperature inside the material increases with respect to time according to equation 22 :

$$
T(z, t)-T_{\infty}=\frac{2 A \Phi}{k} \sqrt{\alpha t} \operatorname{ierfc}\left(\frac{z}{2 \sqrt{\alpha t}}\right)
$$


Table 2: Ablation Onsest for the Different Materials Considered in This Study

\begin{tabular}{llllll}
\hline Material & Forsterite & Al2024 - T3 & Ti 6Al-4V & Carbon Fiber & Unit \\
\hline$(\Phi \sqrt{\tau})_{\text {Threshold }}$ & $0.106 \mathrm{E}+8$ & $1.607 \mathrm{E}+8$ & $0.58 \mathrm{E}+8$ & $0.45 \mathrm{E}+8$ & $\mathrm{~W} \mathrm{~m}^{-2} s^{1 / 2}$ \\
\hline
\end{tabular}

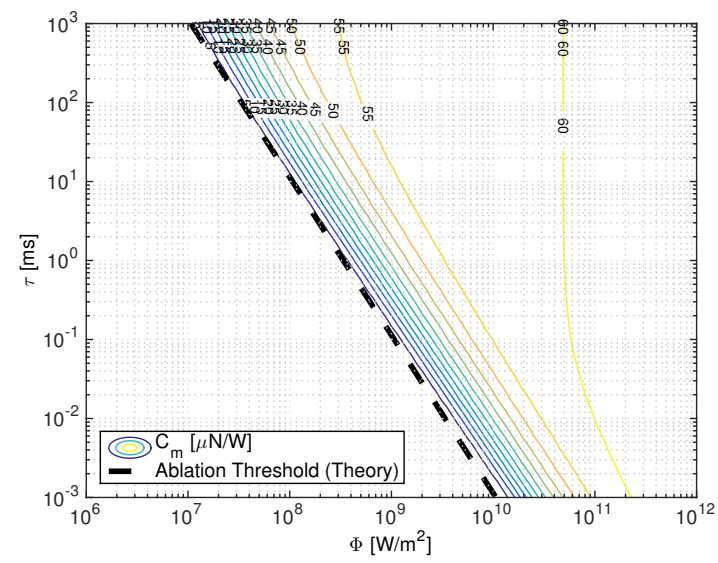

Figure 5: Thrust coupling coefficient as a function of the mean heating time $\tau$ and the optical flux for Forsterite

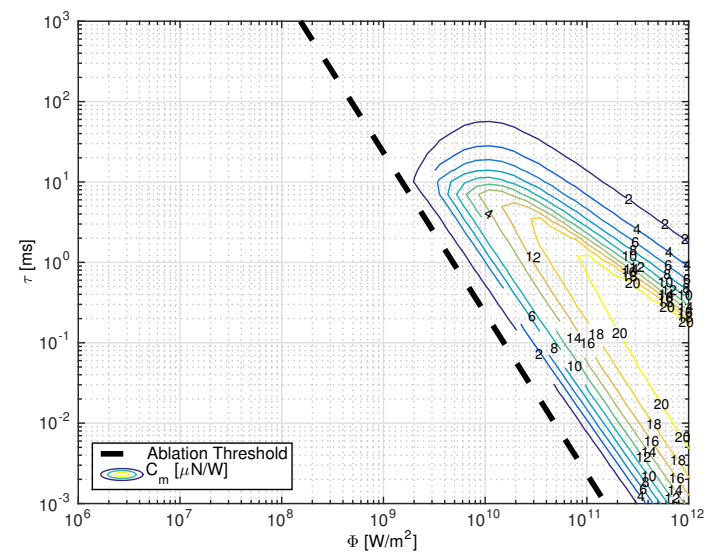

Figure 6: Thrust coupling coefficient as a function of the mean heating time $\tau$ and the optical flux for a $1 \mathrm{~mm}$ panel of Al 2024-T3

Using the the reference temperature as a first approximation and inverting this relation in $\mathrm{z}=0$ allows to compute the ablation threshold as a function of $\Phi \sqrt{\tau}$ :

$$
(\Phi \sqrt{\tau})_{\text {Threshold }} \approx \sqrt{\frac{\pi}{4}} \frac{\Gamma \Delta T_{\text {ref }}}{A}
$$

In which $\Gamma$ is the thermal inertial of the material $\Gamma=\sqrt{\rho c_{l} k}$.

The ablation threshold was computed for the different materials in table 2 and is represented by the dashed black line on figures 5 and 6 and appears to be consistent with the result of the numerical model. The thickness reduction with the recession of the ablation front was also integrated together with Eq.(13) during the computations in the case of the thin aluminium panel and therefore a higher limit on the exposition time as a function of the flux is also visible in figure 6. It corresponds to the time required to melt through the $1 \mathrm{~mm}$ panel. From this figure, one can also see that the region of high efficiency only exists for a combination of very short exposition time and high fluxes. This is a direct consequence of the finite thickness of the material and supports the use of a pulsed laser system in the case of orbital debris rather than a CW laser system.

\section{Pulsed Laser Model for Orbital Debris}

In the case of space debris, we saw that the use of CW lasers to perform the deflection action is unsuitable because the debris components generally possess a finite thickness (typically less than $1 \mathrm{~mm}$ ) and the formation of molten bubbles in the evaporated cloud also needs to be avoided. Moreover, with a pulsed laser system, the duration of the pulses can be chosen to be much lower than the exposition time. In that case, the pulsed laser system will be insensitive to the relative motion of the debris with respect to the laser beam. The model developed in the previous section can be directly applied to the case of a pulsed laser by simply substituting the pulse duration in $\tau$. Using the ablation threshold of Eq.(23), a non-dimensional flux variable can also be formed from the parameters of the problem :

$$
\hat{\Phi}=A \Phi \tau^{1 / 2}\left(\frac{\pi}{4}\right)^{-1 / 2} \Gamma^{-1} \Delta T_{\mathrm{ref}}^{-1}
$$

The results of the model for the different materials are plotted as a function of the non-dimensional flux in figure 7 and compare well with experimental data. Both data sets were obtained by Rosen who studied the coupling on Al 2024-T3 [13] and Ti 6Al-4V alloys [10] using a XeF pulsed laser with $500 \mathrm{~ns}$ pulses.

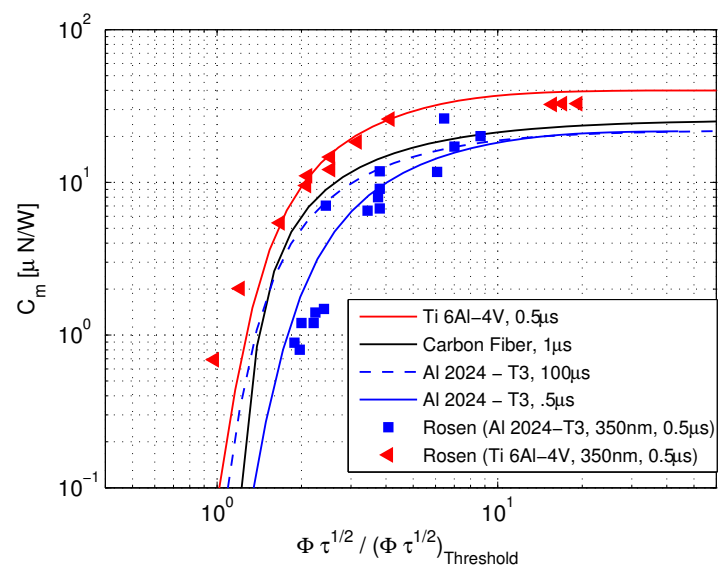

Figure 7: Thrust coupling as a function of $\hat{\Phi}$ for several materials found on space debris

\section{Contamination Model}

The contamination model was adapted from the work of Kahle[1]. From the surface temperature $\mathrm{T}_{s}$, the density $\rho_{e}$ and the velocity $v_{e}$ are computed on the edge of the Knudsen 
layer where the flow reaches the speed of sound in Vacuum. The model assumes two different flow regimes in the near field and in the far field: in the near field. According to Kahle, the density at an arbitrary distance $r$ from the reservoir and angle $\beta$ measured from the local surface normal is approximately given in the continuum flow regime as :

$$
\rho(r, \beta)=\rho_{e} A_{p} \frac{\phi^{2}}{(2 r+\phi)^{2}}\left[\cos \left(\frac{\pi \beta}{2 \beta_{\max }}\right)\right]^{\frac{2}{\gamma-1}}
$$

The jet constant $\mathrm{A}_{p}$ and the limiting expansion angle $\beta_{\max }$ are assumed as equal to 0.345 and 130.45 degrees respectively. The stagnation pressure $p_{0}$ and density $\rho_{0}$ can easily be computed using the isentropic relation:

$$
\begin{aligned}
& \frac{\rho_{e}}{\rho_{0}}=\left(1+\frac{\gamma-1}{2}\right)^{\frac{-1}{\gamma-1}} \\
& \frac{p_{e}}{p_{0}}=\left(1+\frac{\gamma-1}{2}\right)^{\frac{-\gamma}{\gamma-1}}
\end{aligned}
$$

The Mach number $\mathrm{M}$, pressure $\mathrm{p}$ and the velocity in the continuum regime can be computed considering an isentropic expansion of the plume:

$$
\begin{aligned}
M^{2} & =\frac{2}{\gamma-1}\left[{\frac{\rho}{\rho_{0}}}^{1-\gamma}-1\right] \\
\frac{p}{p_{0}} & =\left(1+\frac{\gamma-1}{2} M^{2}\right)^{\frac{-\gamma}{\gamma-1}} \\
v & =\sqrt{\frac{\gamma M^{2} p}{\rho}}
\end{aligned}
$$

The transition from the continuum regime to the free molecular regime happens when the distances between the molecules becomes too large for them to interact. In his simplified model, Kahle proposes to use a sudden transition when the mean free path of the molecules $l_{\mathrm{mfp}}$ become larger than the beam diameter:

$$
l_{\mathrm{mfp}}=\frac{k T}{p \sqrt{24} \pi r_{\mathrm{mole}}^{2}}>\phi
$$

with the molecular radius $\mathrm{r}_{\text {mole }}$ estimated around $2 \cdot 10^{-10} \mathrm{~m}$. Once in the free molecular regime, the assumption is that the velocity becomes constant while the density still decreases with respect to the inverse of the quadratic distance to the spot. The contamination model then assumes that only a fraction $x_{s}$ of the particle impinging on the solar array will stick to it so that the growth of the contamination layer over time can be predicted as

$$
\frac{d m_{A}}{d t}=x_{s} \cdot F(\psi) \cdot v(r, \beta) \cdot \rho(r, \beta)
$$

In the last expression, $\psi$ represents the angle between the normal to the solar panels and the impingement direction of the plume. The view function $\mathrm{F}$ is defined as following:

$$
\begin{aligned}
F & =\cos (\psi) & \text { if }-\frac{\pi}{2}<\psi<\frac{\pi}{2} \\
& =0 & \text { otherwise }
\end{aligned}
$$

Assuming the arrays are pointed towards the sun and the thrust manoeuvre is acted in the tangential direction with respect to the trajectory of the asteroid with the spacecraft remaining in the same orbital plane during the operations, we have:

$$
\cos (\psi-\beta)=-\frac{h e}{a\left(1-e^{2}\right) V} \sin (\theta)
$$

In which $\mathrm{h}$ is the angular momentum, e the eccentricity of the orbit, $\mathrm{V}$ the velocity of the spacecraft and $\theta$ the true anomaly. Finally, a degradation factor $\chi$ can be computed using the Beer-Lambert-Bougier law:

$$
\chi(t)=\exp \left(-\alpha m_{A}(t)\right)
$$

where $\alpha$ is the mass attenuation coefficient which is about $10^{4} \mathrm{~cm}^{2} / g$ for Forsterite [1]. From experimental investigations, we also found that this contamination model predicts correctly the contamination level with a sticking coefficient $x_{s}$ of 0.5 [8]. Over the course of the deflection action a contamination layer will grow on the solar arrays with the degradation factor decreasing slowly from an initial value of 1. This will reduce over time the available input power to the laser system.

\section{UNCOOPERATIVE TARgET REDIRECT Missions}

Several scenarios are considered in order to modify the trajectory of an asteroid or space debris. The orbital trajectory of (2010 KJ37), which is a 56m diameter AMOR asteroid, is considered for this benchmark. (2010 KJ37) has an estimated mass of $2.4 \mathrm{E}+8 \mathrm{kgs}$ and has several low probability impacts with the Earth over the next decades. For the formation option, the mass and diameter were virtually increased to $100 \mathrm{~m}$ and $1.37 \mathrm{E}+9 \mathrm{kgs}$ respectively. Last but not least, we also investigate the case where the target is a large $(>1 \mathrm{~T})$ debris object in LEO.

\section{Asteroid 2010 KJ37}

Asteroid (2010 KJ37) is comparable in size with the asteroid that caused the Tugunska event in 1908 and is thus representative of near-future threats requiring potential mitigation actions to be taken. Two scenarios are investigated in this part: one with the original dimensions of the asteroid and another one with a $100 \mathrm{~m}$ diameter and $1.37 \mathrm{E}+9 \mathrm{kgs}$. The orbital elements were retrieved from the JPL Horizons risk list and slightly modified to form a virtual impact with the Earth in 2036:

Table 3: Orbital Elements for asteroid 2010 KJ37

\begin{tabular}{llll}
\hline Orbital Elements & Symbol & Value (Unmodified) & Value (VI) \\
\hline Semi-major axis & $\mathrm{a}$ & $1.102 \mathrm{AU}$ & $1.102 \mathrm{AU}$ \\
eccentricity & $\mathrm{e}$ & 0.058 & 0.088 \\
inclination & $\mathrm{i}$ & $11.28 \mathrm{deg}$. & $0 \mathrm{deg}$. \\
ascending node & $\Omega$ & $236.43 \mathrm{deg}$. & $236.43 \mathrm{deg}$. \\
argument of perigee & $\omega$ & $99.278 \mathrm{deg}$. & $99.278 \mathrm{deg}$. \\
True anomaly $\left(\mathrm{t}_{0}\right)$ & $\theta$ & $270.978 \mathrm{deg}$. & $270.978 \mathrm{deg}$. \\
\hline
\end{tabular}

The orbit of $2010 \mathrm{KJ} 37$ is represented in figure $8 \mathrm{a}$. Using chemical propellant, the best transfer option would require a $\Delta v$ of $6.5 \mathrm{~km} / \mathrm{s}$ as indicated in figure $8 \mathrm{~b}$.

Lacking better information, the rotation rate was assumed from the formula

$$
\omega_{\max }=\sqrt{\frac{4}{3} \pi \rho G}
$$




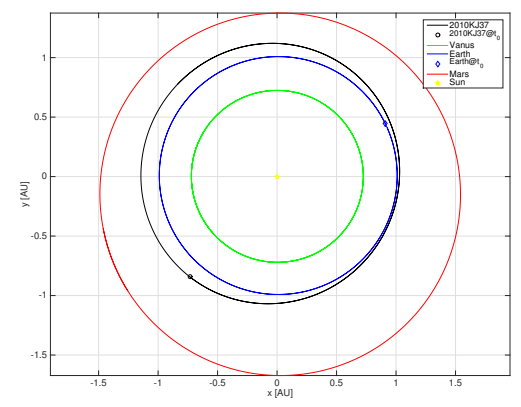

(a) Orbit of asteroid $2010 \mathrm{KJ} 37$

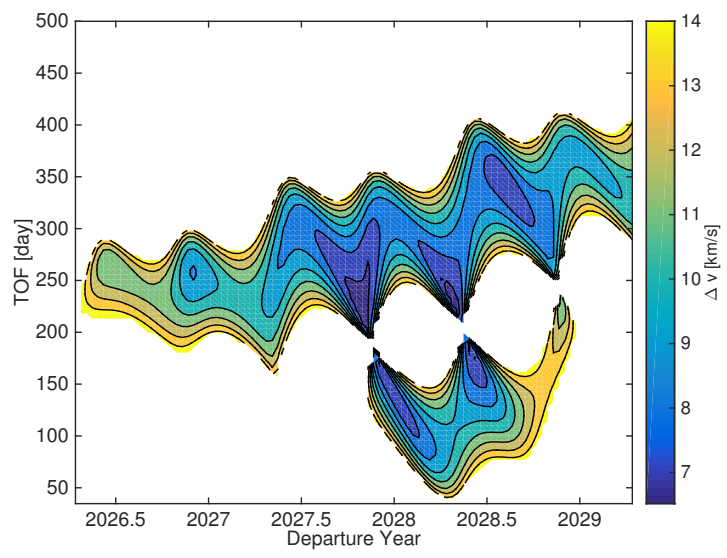

(b) Impulsive transfer cost as a function of the departure date and ToF

Figure 8: Orbit and transfer windows to asteroid $2010 \mathrm{KJ} 37$

which gives the spin limit for asteroids larger than 100m and thus represents an approximation in the smaller case. In a real deflection scenario, an initial observation phase would allow to reduce the uncertainty on the different properties of the target. An estimate of the local surface velocity is then obtained by computing the product between the rotation speed and the asteroid radius, which gives a velocity of the ablated surface relative to the laser beam $v_{\text {rel }}$ that varies between $23.9 \mathrm{~mm} / \mathrm{s}$ for the $56 \mathrm{~m}$ case and $42.9 \mathrm{~mm} / \mathrm{s}$ for the $100 \mathrm{~m}$ case.

The output power of the laser is assumed to vary with respect to the square distance to the sun and will decrease over the lifetime due to contamination issues:

$$
P_{\mathrm{out}}\left(r_{\mathrm{AU}}, t\right)=\chi(t) \frac{P_{\mathrm{out}}\left(1, t_{0}\right)}{r_{\mathrm{AU}}^{2}}
$$

For a given amount of nominal output power at $1 \mathrm{AU}$ $P_{\text {out }}\left(1, t_{0}\right)$ and a given optics able to focus the laser beam on a certain beam diameter $\phi$, our laser-matter interaction model returns the thrust generated and the mass flow rate impinging on the solar arrays, from which the growth of the contamination layer can be computed. The acceleration $\mathrm{a}_{t}$, which is imparted in the tangential direction, is computed from the thrust by dividing by the asteroid mass. The modified trajectory is computed by integrating Gauss' planetary equation:

$$
\begin{aligned}
\frac{d a}{d t} & =\frac{2 a^{2} V}{\mu} a_{t} \\
\frac{d e}{d t} & =\frac{2(e+\cos (\theta))}{V} a_{t} \\
\frac{d \Omega}{d t} & =0 \\
\frac{d \omega}{d t} & =\frac{2}{e V} \sin (\theta) a_{t} \\
\frac{d i}{d t} & =0 \\
\frac{d \theta}{d t} & =\frac{h}{r^{2}}-\frac{2 \sin (\theta)}{e V} a_{t}
\end{aligned}
$$

Where $\mathrm{V}$ is the velocity of the NEO. Note that for large asteroids and a small hovering distance, our model also allows to include the tugging effect of the spacecraft on the asteroid but this effect was not considered in the present study as the tugging force is about 3 orders of magnitude lower than the one due to laser ablation. Moreover, the correction manoeuvre on the spacecraft itself will induce an acceleration on the asteroid of the same order of magnitude as the tugging acceleration but in the opposite direction. Because the perturbed orbit is close to the original one, the achieved deviation $\delta \mathbf{r}$ can be computed from the variation of the orbital parameters at the expected minimum orbit interception distance (MOID).

$$
\begin{aligned}
\delta r_{r} & \approx \frac{r}{a} \delta a+\frac{a e \sin (\theta)}{\nu} \delta M-a \cos (\theta) \delta e \\
\delta r_{\theta} & \approx \frac{r}{\nu^{3}}(1+e \cos (\theta))^{2} \delta M+r \delta \omega \\
& +\frac{r \sin (\theta)}{\nu^{2}}(2+e \cos (\theta)) \delta e+r \cos (i) \delta \Omega \\
\delta r_{h} & \approx r\left(\sin \left(\theta^{*}\right) \delta i-\cos \left(\theta^{*}\right) \sin (i) \delta \Omega\right)
\end{aligned}
$$

in which $\nu=\sqrt{1-e^{2}}$. From the deflection $\delta \mathbf{r}$ the impact parameter $b$ at the time of the MOID can be computed (see Fig.9a where $\mathbf{V}_{\text {NEO }}$ is the velocity of the deviated asteroid with respect to the Earth). The impact plane can be defined as the plane centered in the Earth and perpendicular to the velocity vector of the undeviated asteroid with respect to the Earth, $\mathbf{U}_{\mathbf{N E O}}$, at the time of the impact (see Fig.9b where $\mathbf{v}_{\mathbf{E}}$ is the velocity of the Earth). The deflection vector $\mathbf{x}_{\mathbf{b}}$ in the b-plane coordinates can be expressed as:

$$
\mathbf{x}_{\mathbf{b}}=\left[\begin{array}{lll}
\xi & \eta & \zeta
\end{array}\right]^{\mathbf{T}}=\left[\begin{array}{lll}
\widehat{\xi} & \widehat{\eta} & \widehat{\zeta}
\end{array}\right]^{\mathbf{T}} \delta \mathbf{r}
$$

where:

$$
\widehat{\eta}=\frac{\mathbf{U}_{\mathbf{N E O}}}{U_{N E O}}, \quad \hat{\xi}=\frac{\mathbf{v}_{\mathbf{E}} \wedge \widehat{\eta}}{\left\|\mathbf{v}_{\mathbf{E}} \wedge \widehat{\eta}\right\|}, \quad \hat{\zeta}=\widehat{\xi} \wedge \widehat{\eta}
$$

The impact parameter $b$ is then defined as:

$$
b=\sqrt{\xi^{2}+\zeta^{2}}
$$

The laser-matter interaction model was incorporated in an orbit propagator to determine the deflection achieved for a mission starting the deflection action 5 years prior to the virtual impact. The inputs of this model are the unperturbed 


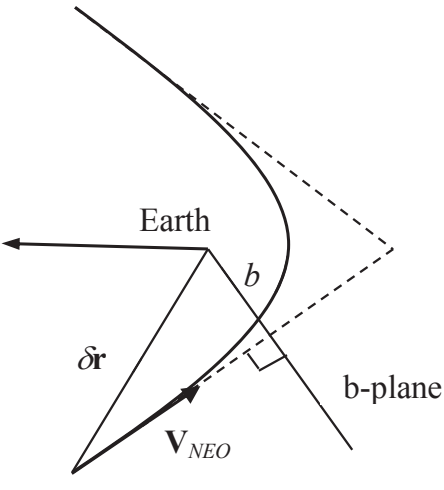

(a)

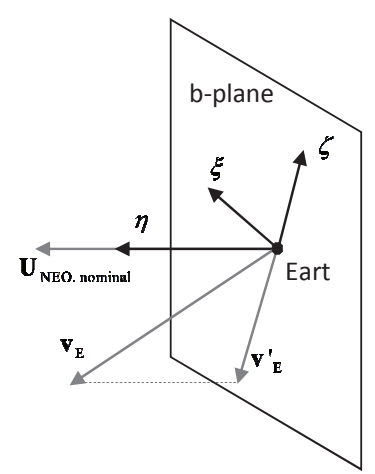

(b)
Figure 9: The impact plane and $b$ parameter

keplerian elements of the virtual impactor, the nominal output power of the laser system (theoretical output power at $1 \mathrm{AU}$ at the start of operations), the beam diameter achieved at the focal length of the focusing optics, the distance between the spacecraft and the target and the duration of the deflection action. The returned value is the miss-distance approximated by the projection of the deflection distance on the target plane (b parameter). Note that the actual value of output power is modulated during the integration to account for the growth of a contamination layer on the solar panels and variation of output power with the square distance to the sun. Figure 10 and figure 11 show the resulting deflection as a function of the nominal output power and focusing ability of the laser system in the case of a $56 \mathrm{~m}, 2.4 \mathrm{E}+08 \mathrm{kgs}$ asteroid and a $100 \mathrm{~m}, 1.37 \mathrm{E}+09 \mathrm{kgs}$ asteroid respectively assuming a spacecraft flying at a $500 \mathrm{~m}$ distance. The results show that

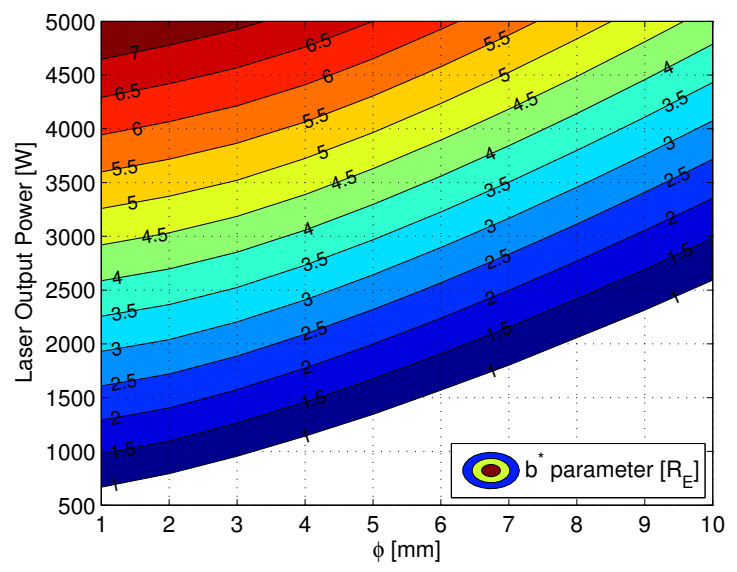

Figure 10: Miss-distance (normalized with respect to the Earth radius) achieved as a function of the available nominal output power at $1 \mathrm{AU}$ and focusing optics for the $56 \mathrm{~m}$ case and 5 years of operation

even a moderate laser system with $2.4 \mathrm{~kW}$ of output power could achieve the deflection within the given warning time and an optics able to focus the laser light on a $3 \mathrm{~mm}$ spot at $500 \mathrm{~m}$ distance. As one would reasonably expects, the results on figure 11 indicate that the power requirement scale approximately with the cube of the asteroid size. For this second case, a single spacecraft with $9.6 \mathrm{~kW}$ of output power could achieve a deflection of about 2 Earth radii within the

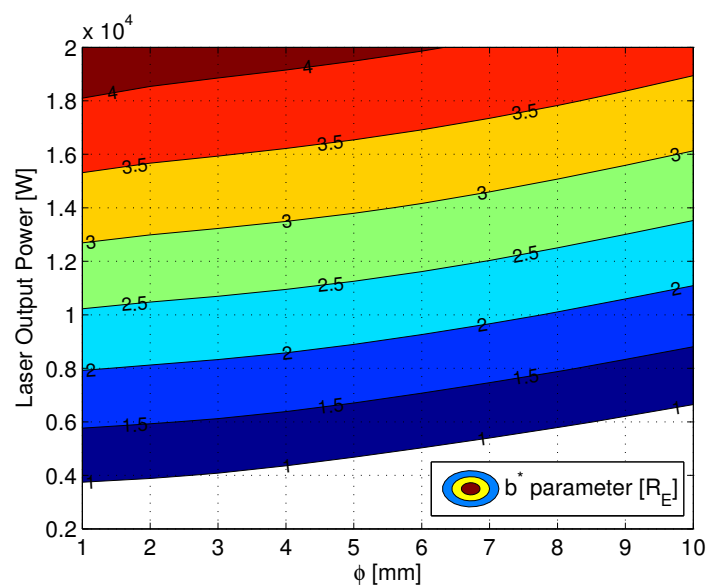

Figure 11: Miss-distance (normalized with respect to the Earth radius) achieved as a function of the available nominal output power at $1 \mathrm{AU}$ and focusing optics for the $100 \mathrm{~m}$ case and 5 years of operation

time imparted and an optics able to focus the laser on a $7 \mathrm{~mm}$ spot. Alternatively, the deflection of the larger asteroid could be achieved by a formation of 4 smaller spacecrafts generating $2.4 \mathrm{~kW}$ of output power each. This option offers a greater redundancy in the case of failure of a single spacecraft and also allow to fit the individual spacecrafts in a mediumclass launcher. If the spacecrafts cannot combine their beams in the far field, the beam diameter required to reach the same efficiency as in the single spacecraft option is reduced to $3 \mathrm{~mm}$. Indeed if the beam diameter was kept constant the flux on the target would not be the same and the mean heating time $\tau$, which depends on this diameter through Eq. (11) would not be the same either.

\section{Large Debris in SSO}

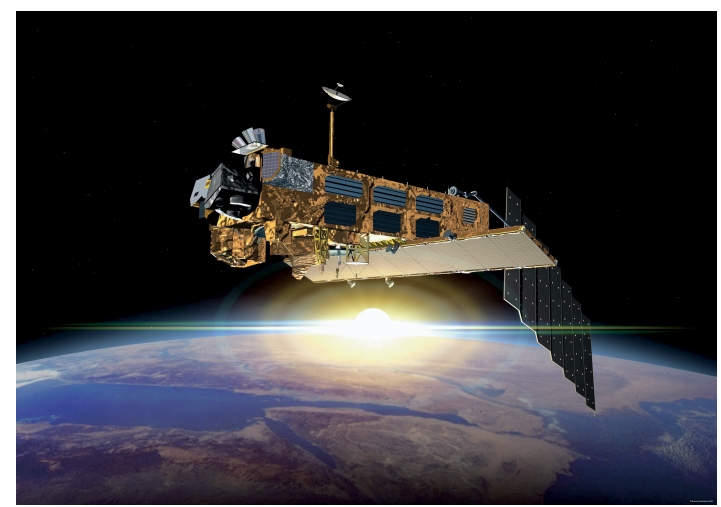

Figure 12: The Envisat Satellite, image courtesy of ESA

We now consider the case of large $(>1 \mathrm{~T})$ man-made debris in sun-synchronous orbit around the Earth. A typical study case is the ESA's 8T envisat satellite (see fig. 12) which was lost in 2012 and is now flying uncontrolled at an altitude of $765 \mathrm{kms}$. The initial orbital elements can be reviewed in table 4: While the eclipses duration are initially identical due to the sun-synchronous nature of the original orbit, this will not remain true while the altitude of the satellite decreases. Therefore, a $\mathrm{J}_{2}$ perturbation term was added to Eq. (38) to correctly predict the drift in the ascending node and argument of perigee over the course of the deflection mission. Eclipses 
Table 4: Orbital Elements of Envisat

\begin{tabular}{lll}
\hline Orbital Elements & Symbol & Value \\
\hline Semi-major axis & $\mathrm{a}$ & $7136 \mathrm{~km}$ \\
eccentricity & $\mathrm{e}$ & 0.0000964 \\
inclination & $\mathrm{i}$ & $98.32 \mathrm{deg}$. \\
ascending node & $\Omega$ & $346.77 \mathrm{deg}$. \\
argument of perigee & $\omega$ & $72.18 \mathrm{deg}$. \\
True anomaly $\left(\mathrm{t}_{0}\right)$ & $\theta$ & $0 \mathrm{deg}$. \\
\hline
\end{tabular}

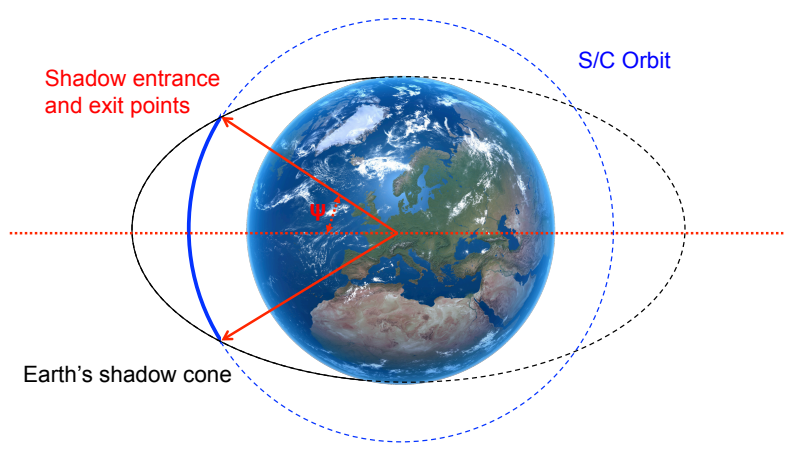

Figure 13: Shadow arc during the spacecraft trajectory (solid)

are accounted in our calculations using a cylindrical shadow approximation which is reasonable for satellites in LEO (the Earth shadow cone being about 106 times longer than its diameter). The assumption is that the laser system only works when the solar panels can receive light from the sun. This configuration is judged to require less mass than an alternative configuration with batteries and a smaller laser system producing the same average power. The eclipses can be calculated by considering the ellipse resulting from the intersection between the shadow cylinder and the orbital plane of the spacecraft first and then calculating the intersections between this ellipse and the spacecraft trajectory.

With reference to figure 13 , for a quasi-circular orbit, the semi-angular distance between the shadow entrance and the shadow exit of the spacecraft $\psi$ can be computed as a function of the orbit radius and the angle between the orbit plane and the sun-earth unit direction vector $\mathrm{s}$ :

$$
\psi=\arcsin \left(\frac{\sqrt{R_{E}^{2}-r_{\mathrm{s} / \mathrm{c}}^{2} \sin ^{2}(\delta)}}{r_{\mathrm{s} / \mathrm{c}} \cos (\delta)}\right)
$$

For a general orbit, a shadow function can be formed as a function of the spacecraft keplerian elements and the sunearth direction vector expressed in the perifocal reference frame[19]. The shadow function is computed as

$$
\begin{aligned}
g(\theta) & =R_{E}^{2}(1+e \cos (\theta))^{2} \\
& +a^{2}\left(1-e^{2}\right)^{2}\left(-s_{x} \cos (\theta)-s_{y} \sin (\theta)\right)-a^{2}\left(1-e^{2}\right)^{2}
\end{aligned}
$$

An eclipse occur when both the function $g(\theta)>0$ and the scalar product $\mathbf{s} \cdot \mathbf{r}_{\mathrm{s} / \mathrm{c}}>\mathbf{0}$. When the spacecraft is under the sunlight, a conservative thrust coupling coefficient of $15 \mu \mathrm{N} / \mathrm{W}$ is assumed, which accounts for the fact that the thrust imparted is only tangential in average. The mass reduction due to the slow vaporization of the target debris is also accounted by the model. An average $\mathrm{I}_{s p}$ of $150 \mathrm{~s}$ is used in the computation. In absolute, this value would depend on the surface temperature reached during the laser pulse. However, for an alluminium alloy, our model indicates that the value only varies from 120 s to 185 s for temperatures ranging between the boiling point $(2790 \mathrm{~K})$ and the critical temperature $(6700 \mathrm{~K})$. The results on figure 14 show the decrease of altitude as a function of the output power of the laser and the duration of the mission. Hence, a laser producing $3 \mathrm{~kW}$ of output power would in theory allow to decrease Envisat's altitude down to $400 \mathrm{~km}$ in less than 500 days.

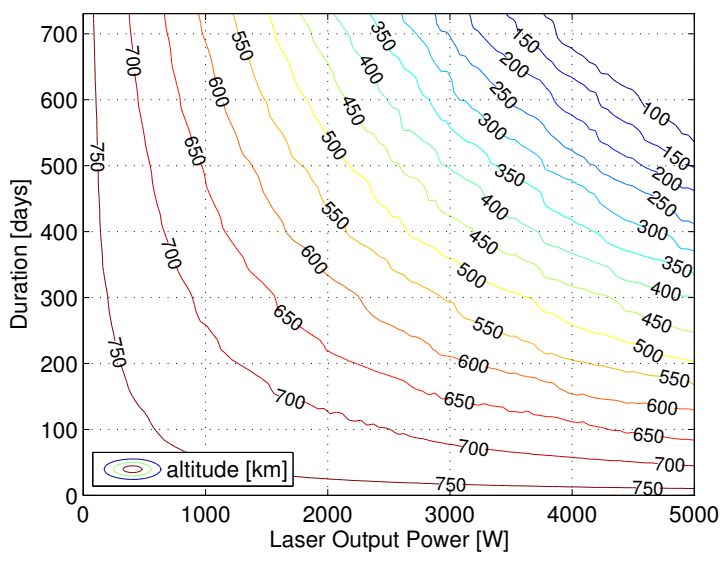

Figure 14: Evolution of the altitude of Envisat as a function of the output power of the laser system and the mission duration

\section{SyStem Design for AN ASTERoID REDIRECT MISSION}

From the results in the last section, it appears that a single spacecraft or a formation of 4 spacecrafts carrying each a laser system producing $2.4 \mathrm{~kW}$ of output power and with an optics able to focus the beam on a $3 \mathrm{~mm}$ spot on the target would be sufficient to achieve the deflection maneuvre in less than 5 years of operation. In this section, we attempt to describe the laser system envisaged to realize this requirement in a power efficient, redundant and scalable way and converts the requirement on the laser system into general requirements on the spacecraft bus. Additionally, we provide in the first point some preliminary guidelines for the conception of the pulsed-laser system required in the debris case.

\section{Laser System}

The mass of the laser system can be estimated by extrapolating previous results obtained during the sysnova study which considered the deflection of a small asteroid using a $860 \mathrm{~W}$ laser [7]. From this study, a mass of $12 \mathrm{kgs}$ per $\mathrm{kW}$ of input power was found for the solid-state laser. The state-of-theart efficiency of diodes is $70 \%$ and is expected to increase to $80 \%$ in the near future [20]. In this study, we considered an efficiency of $55 \%$. The optics considered in the sysnova study consisted in a beam expansion and focusing telescope with a primary mirror of $100 \mathrm{~mm}$, which had an estimated mass of $9.9 \mathrm{kgs}$. The diameter of the primary mirror required 
to achieve a diffraction limited beam of $3 \mathrm{~mm}$ at the focal distance for a $808 \mathrm{~nm}$ laser with a M-squared value of 1.4 is

$$
\phi_{\text {primary }}=M^{2} \frac{4 \lambda f}{\pi \phi_{\text {laser }}}=24 \mathrm{~cm}
$$

Assuming the mass of the optics scales with the square of the diameter of the primary mirror, we obtain a mass of $57 \mathrm{kgs}$. A possible configuration for the laser system is shown in figure 15. It essentially comprises 3 elements:

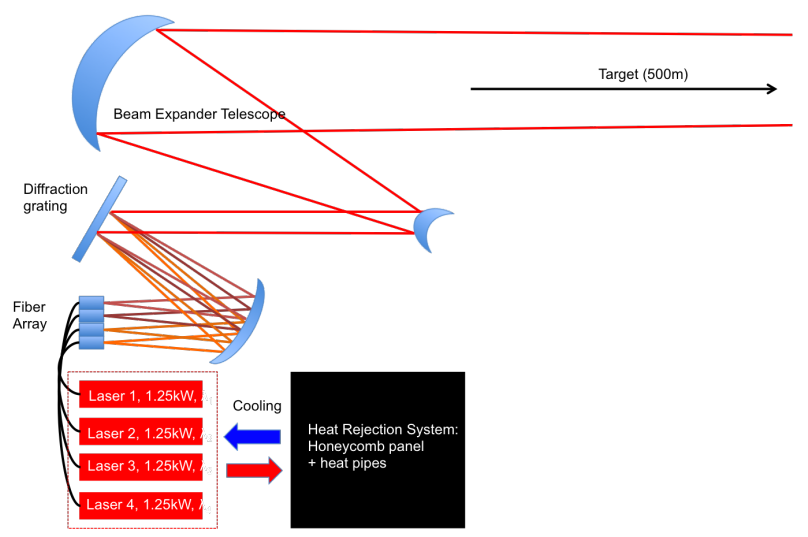

Figure 15: Passive, redundant and up-scalable laser-system design using a spectral beam combining technique and an offaxis beam expansion and focusing telescope

1. The fiber laser chassis which is connected to the electric bus and rejects heat towards the radiators.

2. The spectral beam combination (SBC) module which comprises the fiber array, a transform mirror and the diffraction grating which reflects the different beams with a different angle due to the slightly different wavelength so that the output is a single beam combining the uncoupled incident beams. Lockheed Martin is using this design in its $60 \mathrm{~kW}$ laser for the HEL-MD.

3. An off-axis beam expansion and focusing telescope which allows focusing the laser beam on a $5 \mathrm{~mm}$ spot at a $500 \mathrm{~m}$ distance.

Note that the use of high reflective surfaces both in the SBC module and for the beam expansion telescope will limit the heat production by absorption of the incident light. On the other hand, the use of spectral beam combination technique provides a passive method to upscale the design to higher power, while allowing an increased redundancy in the laser system. The spectral beam combining has the advantage over coherent arrays of permitting independent modulation of the lasers and not requiring precise wavelength and phase control. In addition, such arrays also allows the separate beams to be overlapped in both the near and far fields without spatial interference. Assuming a combination efficiency of $91 \%$ [21], a global laser system efficiency of $50 \%$ is considered. With additional margins of $20 \%$ both for the optics and the laser system, a total mass of $140 \mathrm{kgs}$ is obtained for the laser payload as can be seen in table 5 .

Note that this design could also serve as the basis for the design of the pulsed laser system employed in the debris case, with the fiber array and SBC module used to pump an actively Q-switched Nd:YAG laser for instance. In order to
Table 5: P/L mass budget

\begin{tabular}{lllll}
\hline Component & number & CBE $(\mathrm{kgs})$ & DMM(kgs) & CBE + DMM(kgs) \\
\hline Laser $(1.25 \mathrm{~kW})$ & 4 & 15 & 3 & 18 \\
Optics & - & 57 & 11 & 68 \\
Total & - & 117 & 23 & 140 \\
\hline
\end{tabular}

have sufficient margin with respect to the ablation threshold ( $\Phi \sqrt{\tau} \approx 1 \mathrm{E}+09$ for instance), a system able to deliver a train of $10 \mathrm{~ns}$ pulses containing an energy per pulse of $100 \mathrm{~J}$ at a repetition rate of $33.3 \mathrm{hz}$ and an optics such that the diffraction limited beam has a diameter of $1 \mathrm{~cm}$ could be considered. Such a system would deliver an average power of $3 \mathrm{~kW}$ on the target. Taking into account the additional optical-to-optical conversion due to the diode-pumping, an E/O efficiency of $35 \%$ seems attainable with current technologies [22], giving a required input power of $8.57 \mathrm{~kW}$. From our model, this would allow to reduce Envisat's altitude down to $400 \mathrm{~km}$ in less than 500 days.

\section{Spacecraft Subsystems}

The spacecraft needs to ensure the transfer from the Earth to the asteroid and provide the necessary power to the laser system in order to carry on the deflection mission successfully. It also needs to maintain a $500 \mathrm{~m}$ hovering distance and reasonable pointing accuracy during the 5 years duration proximity operations. After iterations on the design, a total wet mass of $1322 \mathrm{kgs}$ was found for the spacecraft and the mass breakdown between the different subsystems can be reviewed in table 6 . A $\mathrm{C}_{3}$ escape energy of $2.25 \mathrm{~km}^{2} / \mathrm{s}^{2}$

Table 6: Spacecraft mass budget

\begin{tabular}{llll}
\hline Subsystem & CBE(kgs) & DMM(kgs) & Total(kgs) \\
\hline Payload & 117 & 23 & 140 \\
Avionics & 100 & - & 100 \\
AOCS & 63 & 7 & 70 \\
Power & 78 & 16 & 94 \\
Thermal & 38 & 4 & 42 \\
Propulsion & 70 & 11 & 80 \\
Harness & 45 & - & 66 \\
Structure & 128 & - & 187 \\
\hline Dry Mass & 639 & 140 & $\mathbf{7 7 9}$ \\
System Mass Margin \\
Dry Total & & $20 \%$ & 157 \\
\hline Propellant & & & $\mathbf{9 3 6}$ \\
Wet Mass & & & 405 \\
\hline
\end{tabular}

was assumed with the remaining of the transfer completed with a low-thrust trajectory using Snecma's PPS-5000 hall effect thruster which has an Isp of $2300 \mathrm{~s}$ and requires $5 \mathrm{~kW}$ of input power (which are available during the transfer phase). A combination with 2 thrusters (with only 1 working at a time) and 2 PPU/TSU units is considered for redundancy against the failure of a single engine or the high voltage power converter. Lacking better information, a remaining transfer $\Delta v$ of $6 \mathrm{~km} / \mathrm{s}$ was considered in the computations, with an additional safety margin of 30\% (making it a total transfer $\Delta v$ of $7.8 \mathrm{~km} / \mathrm{s}$ using the low-thrust engine and $1.5 \mathrm{~km} / \mathrm{s}$ from the launcher). One clear advantage of the low-thrust option is that it makes use the large available power during the transfer phase, which would otherwise remain unused since the laser only starts operating after rendez-vous with the asteroid. Triple junction GaAs solar cells from Azurspace are considered for the solar arrays which are made of two wings of $10.3 \mathrm{~m}^{2}$ each. Considering a packing efficiency of $85 \%$ 
and an electric conversion efficiency of $26.6 \%{ }^{2}$ (accounting for the damage from the radiations in the interplanetary medium and the transmission of a coverglass with thickness of $100 \mu \mathrm{m})$, this should provide about $6.43 \mathrm{~kW}$ at $1 \mathrm{AU}$. The AOCS consists of 4 RSI 45-745/60 reaction wheels placed in a redundant tetrahedral configuration. In addition to these a combination of $1 \mathrm{~N}$ hydrazine thrusters are needed to facilitate orbit insertion and proximity manoeuvres required to compensate for the perturbations coming from the solar radiation pressure on the solar wings. The other perturbations, coming from the gravity of the asteroid and the laser recoil force are assumed to be compensated by the electric propulsion system and require only a negligible amount of Xenon propellant. The AOCS also includes 2 star trackers, a LIDAR and wide angle camera for navigation.

According to figure 16, a medium class launcher such as Soyuz or Falcon 9 could put the spacecraft on its interplanetary course [23][24]. Launch capabilities (in kgs) for interplanetary missions depend on the characteristic energy $\mathrm{C}_{3}$ which is a measure of the excess specific energy over that required to just barely escape from the Earth.

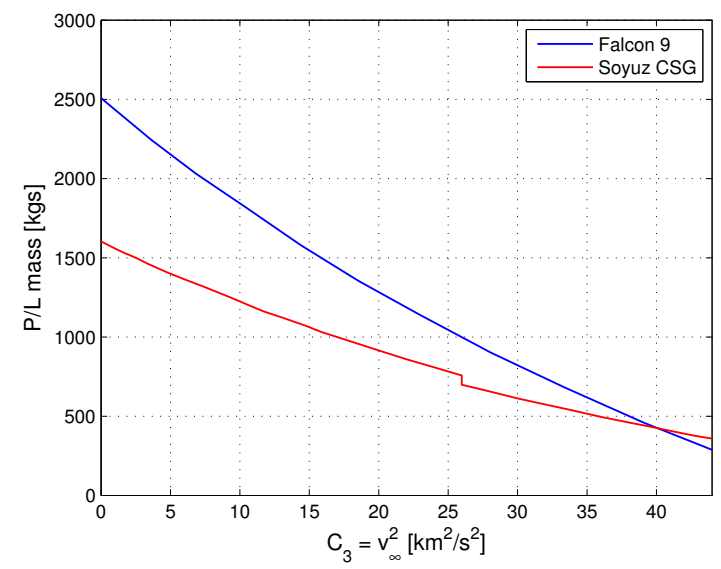

Figure 16: Interplanetary launch capabilities as a function of the $\mathrm{C}_{3}$ energy for Falcon 9 and Soyuz CSG

For comparison, Nasa's Dawn spacecraft, which recently visited the dwarf planet Ceres using 3 NSTAR gridded ion thrusters and achieved a record cumulated $\Delta v$ of $14 \mathrm{~km} / \mathrm{s}$, had a wet mass of $1240 \mathrm{kgs}$ with $425 \mathrm{kgs}$ of Xenon propellant, a dry spacecraft mass of $815 \mathrm{kgs}$ and a solar array of $36.4 \mathrm{~m}^{2}$ able to deliver $10 \mathrm{~kW}$ at $1 \mathrm{AU}$.

\section{Conclusion}

In conclusion, we developed a new model of the lasermatter interaction applied to targets made of asteroid or thin debris materials. This model can predict the thrust coupling coefficient attained in theory by CW or pulsed laser systems on a given material. Comparisons with published data for aluminium and titaninum alloys show that the thrust coupling predicted is in good agreement with the experiments. Integrating these models within an orbit propagator, we also

\footnotetext{
${ }^{2}$ which is later multiplied by the contamination factor $\chi$ during the compu-
} tations observed the influence for a given deflection scenario of the output power and focusing optics of the laser system assuming a 5 years duration for the mission. Additionally, we investigated the ability of a pulsed-laser system to de-orbit Envisat as a function of the mission duration and the ouput power produced by the laser. The results on the asteroid case indicates that a medium launcher could be used to inject a $1.3 \mathrm{~T}$ spacecraft or a fleet of such spacecrafts to deflect asteroids ranging from 50 to $100 \mathrm{~m}$ in diameter. Our preliminary results for the debris case also indicates that an actively Qswitched diode-pumped solid state laser (DPSSL) with $3 \mathrm{~kW}$ of output power would reduce the altitude of Envisat in less than 500 days. Future works will assess the ability of such a mission to fit in a Vega launcher and the possibility to deorbit multiple large debris in SSO with such a system.

\section{ACKNOWLEDGMENTS}

This work is funded by the European Commission's Framework Programme 7, through the Stardust Marie Curie Initial Training Network, FP7-PEOPLE-2012-ITN, Grant Agreement 317185 .

\section{REFERENCES}

[1] R. Kahle, E. Kührt, G. Hahn, and J. Knollenberg, "Physical limits of solar collectors in deflecting Earththreatening asteroids," Aerospace Science and Technology, vol. 10, pp. 253-263, 2006.

[2] M. Vasile and C. Maddock, "On the deflection of asteroids with mirrors," Celestial Mechanics and Dynamical Astronomy, vol. 107, no. 1, pp. 265-284, May 2010.

[3] M. Vasile, A. Gibbings, I. Watson, and J.-M. Hopkins, "Improved laser ablation model for asteroid deflection," Acta Astronautica, vol. 103, pp. 382-394, 2014.

[4] S.-M. Yoo, Y.-J. Songa, S.-Y. Park, and K.-H. Choi, "Spacecraft formation flying for Earth-crossing object deflections using a power limited laser ablating," $A d$ vances in Space Research, vol. 43, no. 12, pp. 18731889, 2009.

[5] M. Vasile and C. A. Maddock, "Design of a formation of solar pumped lasers for asteroid deflection," Advances in Space Research, vol. 50, no. 7, pp. 891-905, 2012.

[6] F. Zuiani, M. Vasile, and A. Gibbings, "Evidence-based robust design of deflection actions for near earth objects celestial mechanics and dynamical astronomy," Celestial Mechanics and Dynamical Astronomy, vol. 114, no. 1-2, pp. 107-136, 2012.

[7] M. Vasile, A. Gibbings, V. Massimo, J.-P. Sanchez, D. Yarnoz, S. Eckersley, A. Wayman, J. Branco, D. Burns, J.-M. Hopkins, C. Colombo, and C. McInnes, "Light touch2: Effective solutions to asteroid manipulation. sysnova challenge analysis final report," University of Strathclyde, Tech. Rep., 2013.

[8] A. Gibbings, M. Vasile, I. Watson, J.-M. Hopkins, and D. Burns, "Experimental analysis of laser ablated plumes for asteroid deflection and exploitation," Acta Astronautica, vol. 90, no. 1, pp. 85 - 97, 2013, NEO Planetary Defense: From Threat to Action.

[9] R. Powell, C. Y. Ho, and P. E. Liley, "Thermal conductivity of selected materials," DTIC Document, Tech. Rep., 1966.

[10] D. Rosen, D. Hastings, and G. Weyl, "Coupling of 
pulsed $0.35-\mu \mathrm{m}$ laser radiation to titanium alloys," Journal of applied Physics, vol. 53, no. 8, pp. 5882-5890, 1982.

[11] M. Boivineau, C. Cagran, D. Doytier, V. Eyraud, M.-H. Nadal, B. Wilthan, and G. Pottlacher, "Thermophysical properties of solid and liquid ti-6al-4v (ta6v) alloy," International journal of thermophysics, vol. 27, no. 2, pp. 507-529, 2006.

[12] N. Thiry and M. Vasile, "Recent advances in laser ablation modelling for asteroid deflection methods," in SPIE Optical Engineering + Applications. International Society for Optics and Photonics, 2014, pp. 922 608-922608.

[13] D. Rosen, J. Mitteldorf, G. Kothandaraman, A. Pirri, and E. Pugh, "Coupling of pulsed $0.35-\mu \mathrm{m}$ laser radiation to aluminum alloys," Journal of applied Physics, vol. 53, no. 4, pp. 3190-3200, 1982.

[14] C. Freitag, R. Weber, and T. Graf, "Polarization dependence of laser interaction with carbon fibers and cfrp," Optics express, vol. 22, no. 2, pp. 1474-1479, 2014.

[15] C. J. Knight, "Theoretical modeling of rapid surface vaporization with back pressure," AIAA journal, vol. 17, no. 5, pp. 519-523, 1979.

[16] C. Phipps, M. Birkan, W. Bohn, H.-A. Eckel, H. Horisawa, T. Lippert, M. Michaelis, Y. Rezunkov, A. Sasoh, W. Schall et al., "Review: laser-ablation propulsion," Journal of Propulsion and Power, vol. 26, no. 4, pp. 609-637, 2010.

[17] N. Thiry and M. Vasile, "Deflection of uncooperative targets using laser ablation," in SPIE Optical Engineering + Applications. International Society for Optics and Photonics, 2015, pp. $96160 \mathrm{X}-96$ 160X.

[18] S. I. Anisimov and V. A. Khokhlov, Instabilities in laser-matter interaction. CRC press, 1995.

[19] D. A. Vallado and W. D. McClain, Fundamentals of astrodynamics and applications. Springer Science \& Business Media, 2001, vol. 12.

[20] P. Cramp, J. Wang, S. Das, M. Grimshaw, J. Farmer, M. DeVito, W. Dong, T. Crum, D. Wise, and Y. Feng, "i 360w and $i$ 70\% efficient gaas-based diode lasers," DTIC Document, Tech. Rep., 2005.

[21] D. Drachenberg, I. Divliansky, V. Smirnov, G. Venus, and L. Glebov, "High-power spectral beam combining of fiber lasers with ultra high-spectral density by thermal tuning of volume bragg gratings," in SPIE LASE. International Society for Optics and Photonics, 2011, pp. $79141 \mathrm{~F}-79141 \mathrm{~F}$.

[22] C. R. Phipps, "L adroit-a spaceborne ultraviolet laser system for space debris clearing," Acta Astronautica, vol. 104, no. 1, pp. 243-255, 2014.

[23] SpaceX, Falcon 9 Launch Vehicle Payload User's Guide, 2009.

[24] Arianespace, Soyuz from the Guiana Space Centre User's Manual, 2006.

\section{BIOGRAPHY}

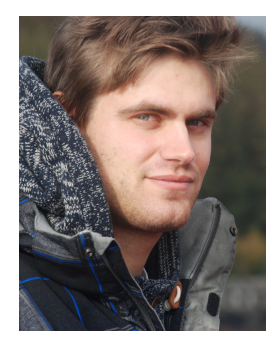

Nicolas Thiry received his M.Sc. degree in Mechanical Engineering from Universite Catholique de Louvain in Belgium and completed a research master in fluid dynamics from the von Karman Institute (NATO/STO) where he was awarded the Belgian government prize for his work on supersonic inlet unstart mechanisms. Prior to this work, he also completed a young graduate trainee in the structure section of ESA's space research and technology centre in the Netherlands (ESA/ESTEC). His current PhD research involves studying new technologies to manipulate uncooperative targets in space and is carried out within the frame of the Stardust FP7 Marie Curie Initial Training Network at the University of Strathclyde, United Kingdom.

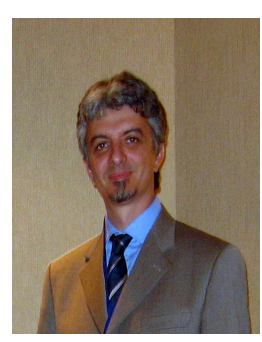

Massimiliano Vasile is the Director of the Advanced Space Concepts Laboratory, and Professor of Space Systems Engineering in the Mechanical \& Aerospace Engineering Department at the University of Strathclyde. Previous to this he was a Senior Lecturer in the Department of Aerospace Engineering and Head of Research for the Space Advanced Research Team at the University of Glasgow. His main research interests are Space Mission Analysis and Design, Computational Optimization, Optimization Under Uncertainty, Multidisciplinary Design, Asteroids, Space Flight Mechanics, and Autonomous Robotic Systems. He is member of the IAF Space Power and Astrodynamics Committees.

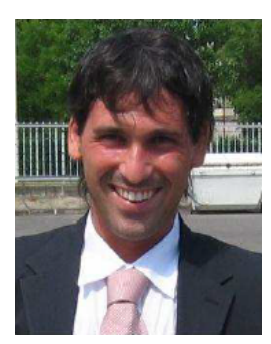

Emanuele Monchieri has gained an extensive experience working on several mission and technology studies, with the focus on Moon and Mars robotic and manned exploration as well as Space Situational Awareness projects among which NEOShield 1 \& 2 and Space Weather related activities. In particular he has been responsible for the identification and assessment of enabling technologies/capabilities for supporting the ESA exploration roadmap, for the design of autonomous, teleoperated, and human-driven surface mobility platforms, and for the assessment of alternative demonstration missions for deflecting an asteroid. 